\title{
The use of gamma rays in studying the homogeneity of the alloy (AL-Co-Ni) reinforced with chromium oxide
}

\author{
Laith Rabih $^{1}$, Raad A. Rasool ${ }^{2}$, Ali K. Hussein ${ }^{3}$ \\ ${ }^{1,2 * 3}$ Physics Department, College of Education for Pure Science, IRAQ \\ E-mail: ${ }^{1}$ laithrabih@uomosul.edu.iq, ${ }^{2,}$ 르.raadrasool@uomosul.edu.iq, ${ }^{3}$ alikhatab@yahoo.com,
}

(Received April 07, 2021; Accepted July 16, 2021; Available online December 01, 2021)

DOI: 10.33899/edusj.2021.129868.1156, @ 2021, College of Education for Pure Science, University of Mosul.

This is an open access article under the CC BY 4.0 license (http://creativecommons.org/licenses/by/4.0/)

\begin{abstract}
This research deals with the preparation number of composite materials by casting the base alloy (AlCo-Ni) by adding chromium oxide $\left(\mathrm{Cr}_{2} \mathrm{O}_{3}\right)$ as reinforcement material, by weight ratios of $(5,10,15$ wt\%) for the base alloy, with heat treatment of the prepared alloys. The basic alloys and composite materials were prepared by using solution casting method and manual mixing method to disperse reinforcement grains in the base alloy floor and with pressure of 5 ton in purpose of forming. The materials were melted and poured into molds and suddenly cooled in cold water, after the molten samples were frozen. The attenuation factor of gamma rays was founded the prepared alloys and their homogeneity was examined by shining a narrow beam of single energy gamma rays emitted from the radiating source $\left({ }^{137} \mathrm{Cs}\right)$ with different energies $(511,662,1173,1284,1333) \mathrm{keV}$ respectively, gamma ray system (UCS-20) was being used which bounded to $\mathrm{NaI}(\mathrm{Tl})$ scintillation detector. The homogeneity of base alloy and composite material was determined by studying the contrast of gamma ray intensity, the linear attenuation coefficient at seven different locations at any samples and the percentage standard deviation. The results showed that the highest value of the linear attenuation coefficient was $0.252 \mathrm{~mm}^{-1}$ for sample $\left[5 \% \mathrm{Cr}_{2} \mathrm{O}_{3}+\mathrm{Al}+\mathrm{Co}+\mathrm{Ni}\right]$ with the energy $(511 \mathrm{keV})$ and that the lowest value for the ratio of linear attenuation coefficient was $0.062 \mathrm{~mm}^{-1}$ at the energy of sample $\left[15 \% \mathrm{Cr}_{2} \mathrm{O}_{3}+\mathrm{Al}+\mathrm{Co}+\mathrm{Ni}\right]$ with the energy $(1333 \mathrm{keV})$ indicating that the homogeneity of the sample $\left[15 \% \mathrm{Cr}_{2} \mathrm{O}_{3}+\mathrm{Al}+\mathrm{Co}+\mathrm{Ni}\right]$ at energy $(1333 \mathrm{KeV})$.
\end{abstract}

Keywords: Compounds, homogeneity, gamma rays, chromium oxide, alloy $\left[\mathrm{Cr}_{2} \mathrm{O}_{3}+\mathrm{Al}+\mathrm{Co}+\mathrm{Ni}\right]$

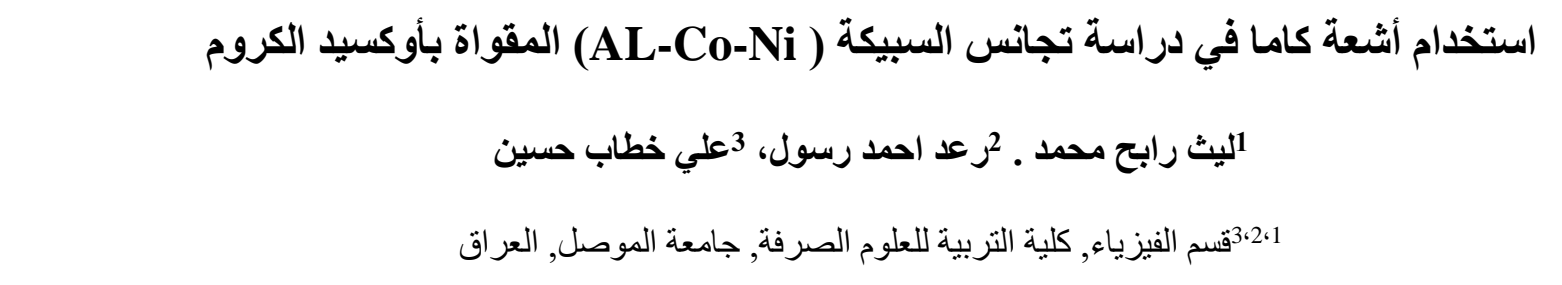

يتتاول البحث تحضير عدد من المواد المتراكبة بطريقة السباكة للسبيكة الأساس (Al-Co-Ni) بإضافة أوكسيد

الكروم (5, 10, 15 wt\%) للسبيكة الأساس، مع إجراء المعالجة الحرارية 
للسبائك المحضرة. حُضرت السبائك الأساسية والمواد المتراكبة معها باستخدام طريقة السباكة المحلولية وطريقة الخلط

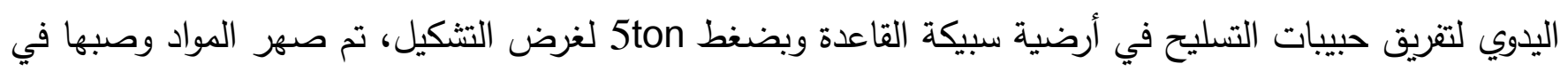
قوالب وتبريدها بشكل مفاجئ في الماء البارد، بعد تجميد العينات المصهورة.

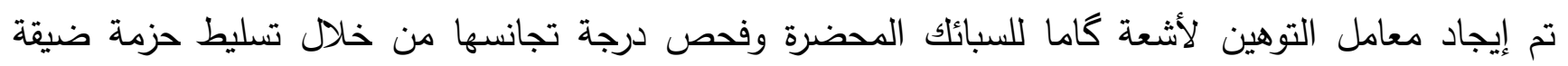

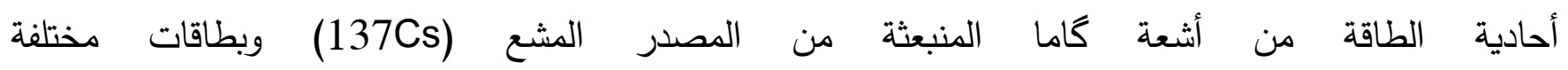
UCS-) (511,662,1173,1284,1333) KeV 20) المرتبطة بالكاشف ألوميضي Nal(TI). حددت درجة تجانس السبيكة الأساس والمواد المتراكبة من خلال دراسة تباين شدة أشعة كاما ومعامل التوهين الخطي في سبع مواقع مختلفة من المادة والنسبة المئوية للإنحراف المعياري.

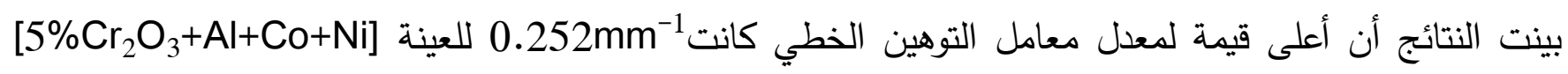

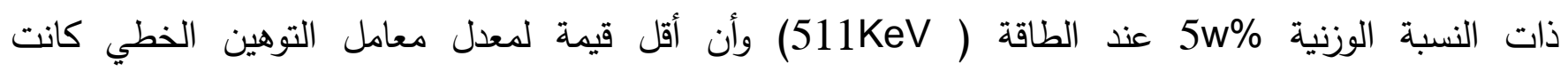
0.062mm² (1333 KeV) عند الطاقة $\left.15 \% \mathrm{Cr}_{2} \mathrm{O}_{3}+\mathrm{Al}+\mathrm{Co}+\mathrm{Ni}\right]$

الكلمات الدالة: المواد المتراكبة, التجانس, اشعة كاما، اوكسيد الكروم, السبيكة [Cr

\section{(لمقدمة}

لقد أدى التطور الصناعي والمدني الى الحاجة لإيجاد مواد ذات مواصفات نوعية عالية من حيث الكلفة وخفة الوزن

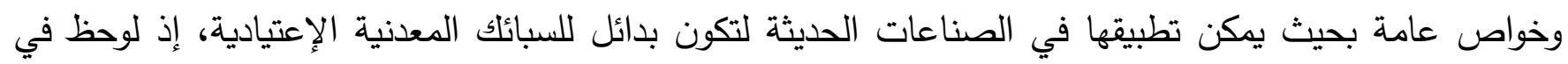
مجال علم المواد وجود إختلاف في خواص تلأك المواد من حيث تحملها لدرجات الحرارة والإجهادات الخارجية والمتانة والصلادة والليونة، وهي خصائص مرغوبة في التطبيقات الصناعية [1,2].

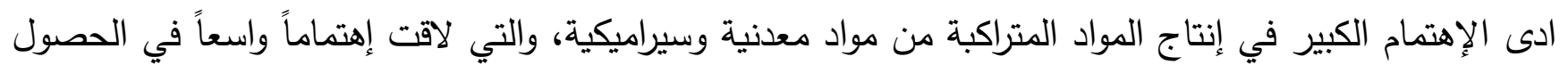

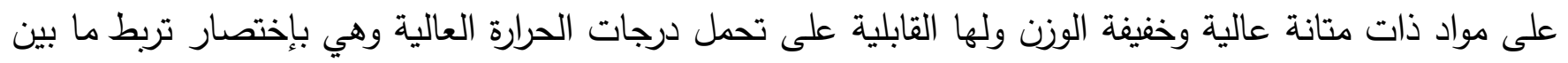

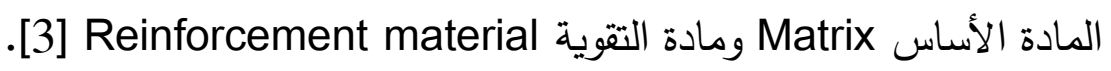
تتضمن السباكة الخلائط الميكانيكية تصنيع منتجات من مساحيق المواد الأولية بدون عمليات صهر وإدماجها معاً وتقويتها بعمليات التلبيد الحراري والتي تعد من أهم التقانات المتبعة في تصنيع المواد المتراكبة [4]. هناك العديد من المواد يمكن استعمالها دروعا واقية من الإشعاع بسبب قدرتها على توهين الإشعاع وإمتصاصها.

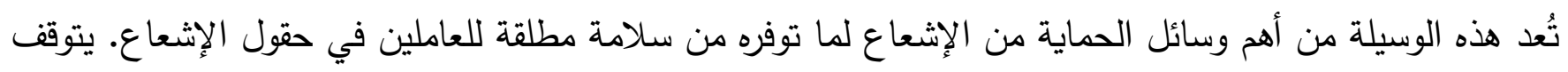

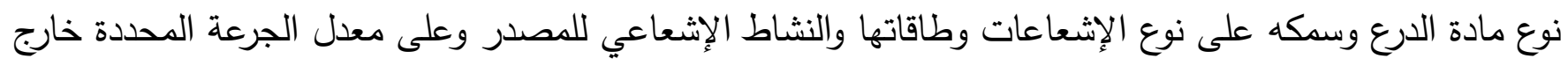


هذا الدرع. تعتمد عملية توهين الأشعة الكهرومغناطيسية على الثكل الهندي للحزمة الإشعاعية من ناحية كونها

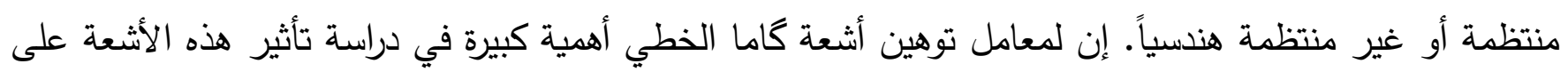

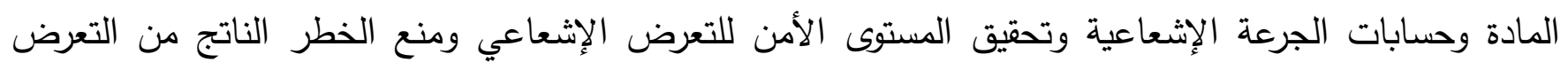

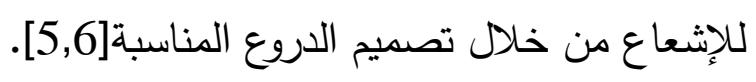
إن إضافة المواد المتراكبة لبعض أنواع السبائك تُظهر تعزيزاً واضحاً في الخصائص الميكانيكية والبنية البلورية [7].

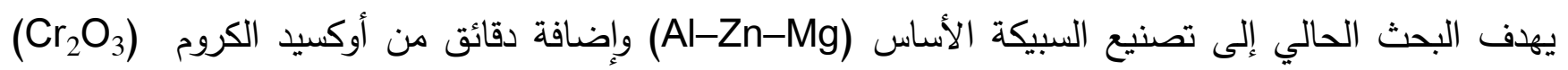

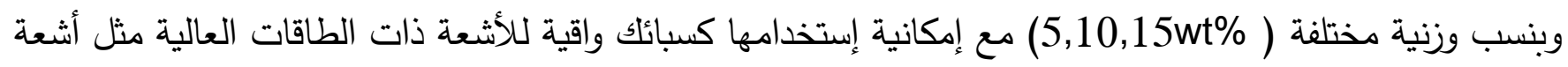
كاما ,حيث تم دراسة هذه السبائك من خلال تسليط أشعة كاما المنبعثة من المصدر المشع (137Cs) وبطاقات

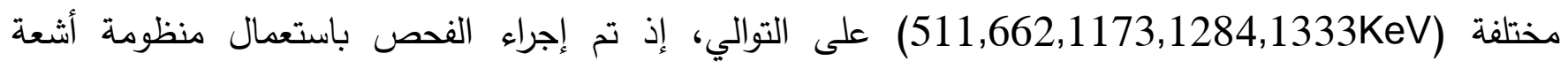
كاما (UCS-20) المرتبطة بالكاشف الوميضي (Tal(TI).

الجانب العملي 1. تحضير السبيكة الأساس حُضرت السبيكة الأساس (Al- 4\%CO- 1\%Ni) بصهر قطعة من الألمنيوم ذات نقاوة ( 99\% ) وبكتلة معلومة في داخل بودقة من معدن الصلب (steel) داخل فرن كهربائي، ولضمان إتمام عملية الإنصهار بالكامل أجريت عملية الصهر في درجة حرارة (850 C C الكوبالت النقي بنسبة وزنية 4wt\% , وتفادياً لحدوث عملية أكسدة تعيق عملية الصهر فقد كانت عملية الغمر سريعة الإنهار جدا مع التحريك المستمر للمنصهر بقضيب من الصلب، ثم أضيفت رقائق من النيكل النقي بنسب وزنية (1wt\%)

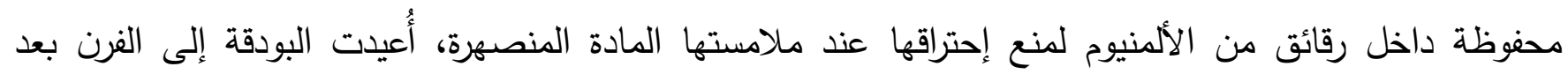
الإنتهاء من عملية الإضافة تلاك، ولتجانس عناصر السبك بشكل تام تكرر مزج المنصهر (3 مرات) , وللمحافظة

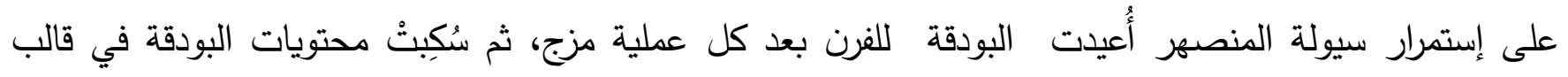

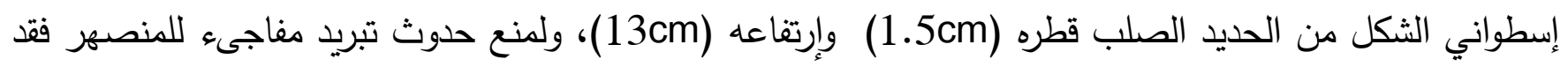
سُخن القالب مسبقاً إلى درجة حرارة (250²C)، وبعد انجماد المنصهر كاملاً تم تبريد العينة بالماء البارد لمنع زيادة مسافة أذرع الثجيرات [8]. ثم تخرج العينة المصبوبة من القالب للحصول على السبيكة الأساس.

2 2. تحضير المادة المتراكبة

حضرت المادة المتراكبة بالسباكة المعدنية، إذ قطعت السبيكة الأساس قطعاً صغيرة لتحديد الوزن المطلوب منها استتادا إلى الكسر الوزني لمادة التقوية، وضعت القطع الموزونة داخل بودقة ووضعت داخل الفرن الكهربائي الذي 
نُظمت درجة حرارته إلى (850º C بعد التأكد من إنصهار السبيكة بشكل تام، أُضيفت دقائق أوكسيد الكروم وبنسب

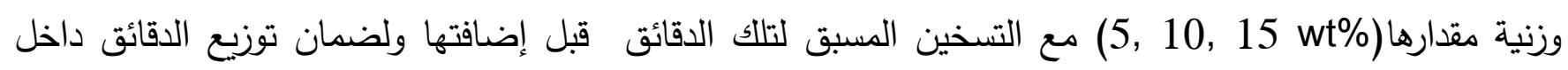

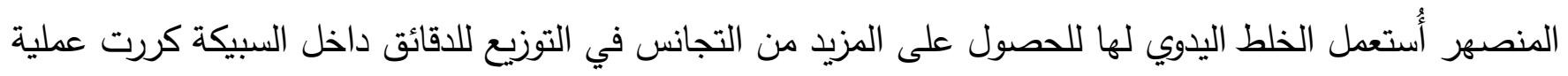

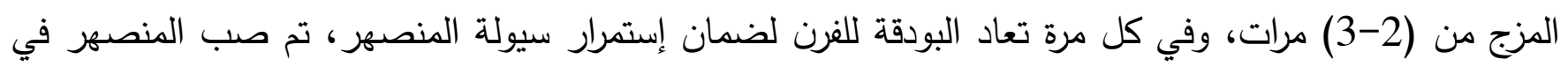

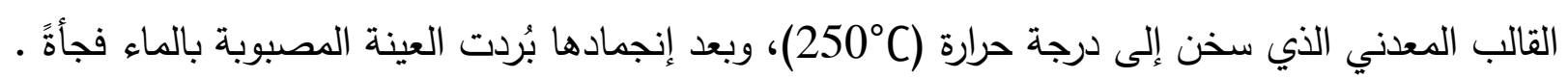
3. المعاملة الحرارية بعد تقطيع العينات بالأبعاد القياسية للسبيكة الاساس والمتراكبة اجريت المعاملة الحرارية المحلولية للعينات في درجة

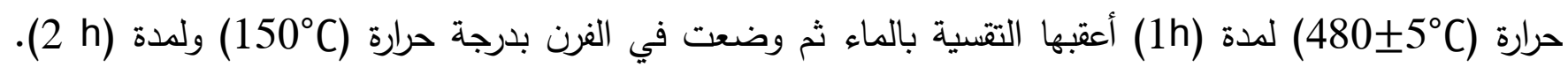
الثكل 1. يمثل صورة للعينات المستخدمة.

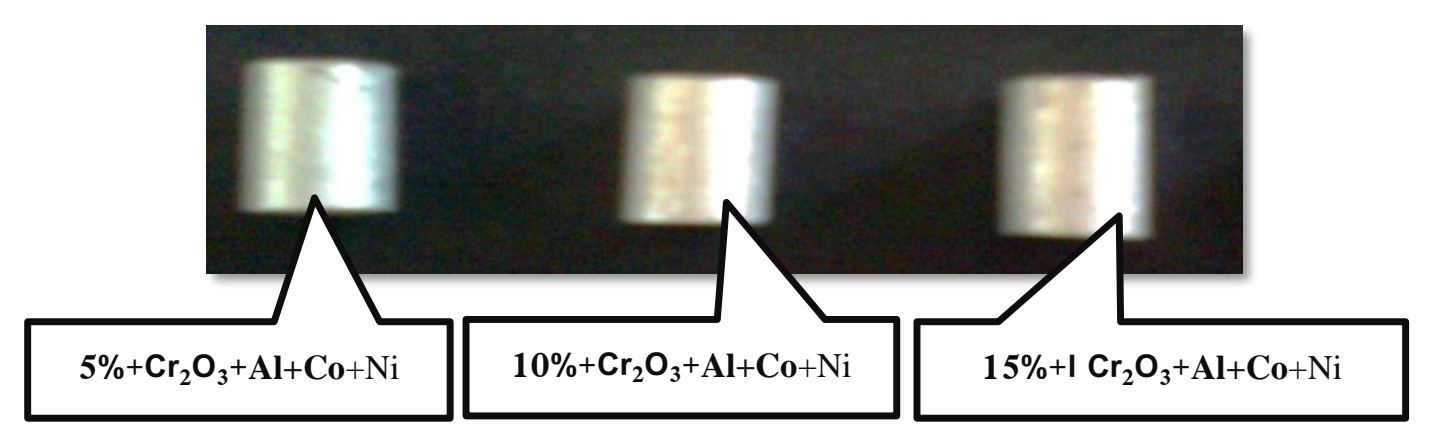

الثكل1. العينات المستخدمة في فحص التجانس

4. منظومة القياس تم إيجاد معامل توهين أشعة كاما للسبائك أعلاه وفحص درجة تجانسها من خلال تسليط أشعة كاما المنبعثة من المصدر المشع (137Cs) وبطاقات مختلفة (511,662,1173,1284,1333keV) على التوالي، إذ اجري دونهي الفحص باستعمال منظومة أشعة كاما UCS-20 المرتبطة بالكاشف الوميضي Nal(TI) ذيطي الأبعاد 2.5cm×3.8cm والموضحة في الثكل 2. تتكون هذه المنظومة من مضخم إبتدائي ومضخم رئيس ومجهز للفولتية ومحلل متعدد القنوات وترتبط هذه المنظومة بجهاز حاسوب لغرض تشغيلها وقراءة قياساتها وتحليل النتائج. 


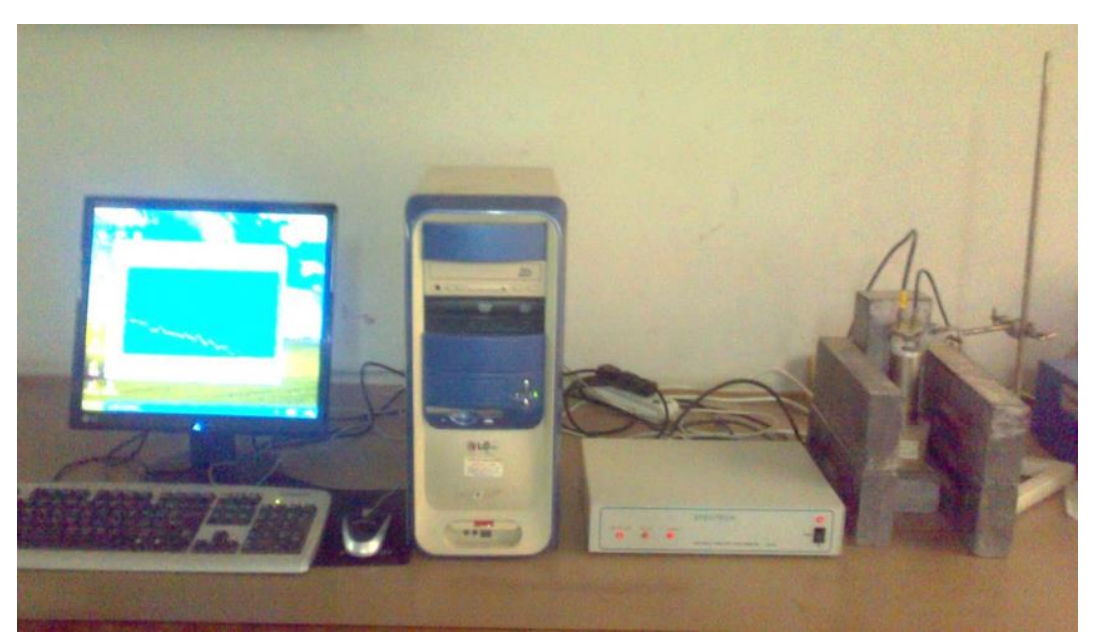

الثكل2. المنظومة الإلكترونية الخاصة لقياس تجانس العينات

أستعملت حزمة ضيقة من أشعة كاما وذللك باستخدام مسدد من الرصاص إسطواني الثكل إرتفاعه 6cm وقطره 4cm ويحتوي على فتحة قطرها 2mm (المؤشرة في الموقع 1) تمر من خلالها الأشعة القادمة من المصدر المشع. يحتوي المسدد على فتحة ضيقة قطرها 8mm (المؤشرة في الموقع 2) توضع بداخلها العينة المراد قياس معامل من فئل

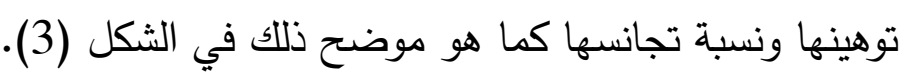

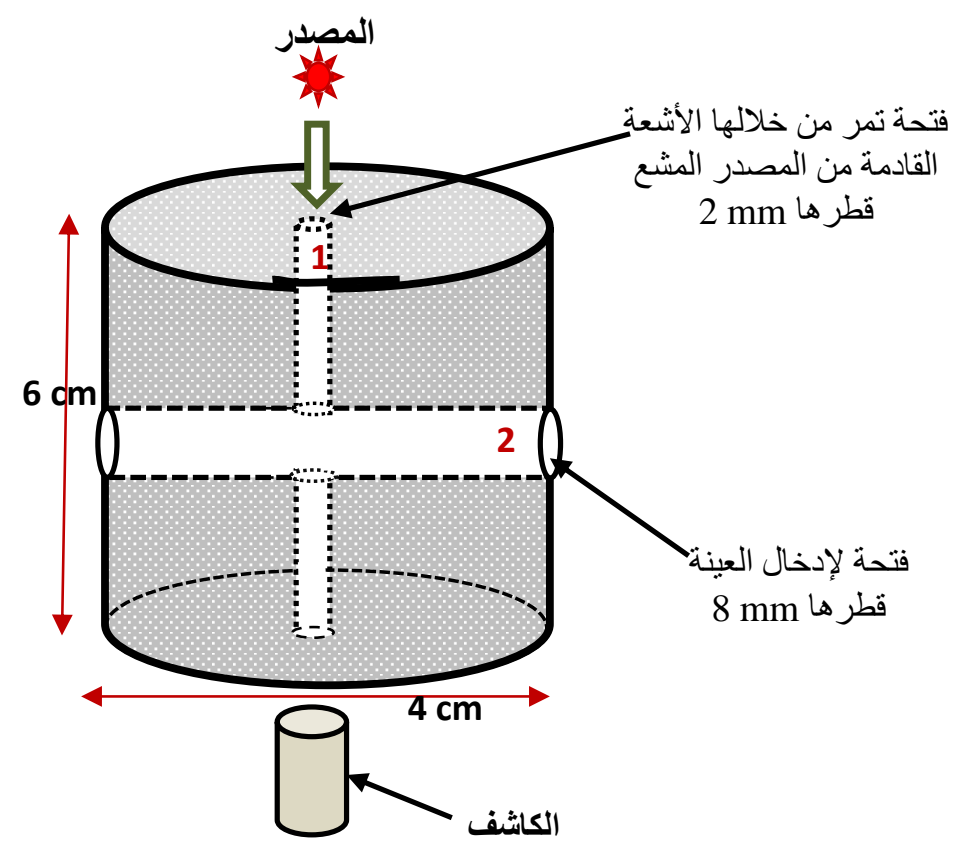

الثكل3. مخطط للمسدد المصوع من الرصاصو الذي يبين طريقة اخز اق اشعة

الحسابات

1. 1. عامل توهين الخطي لأثبعة گاما 
عند سقوط حزمة متوازية من إشعاعات كاما على المادة تتفاعل مع ذرات المادة بإحدى التفاعلات الثلاثة (التأثير

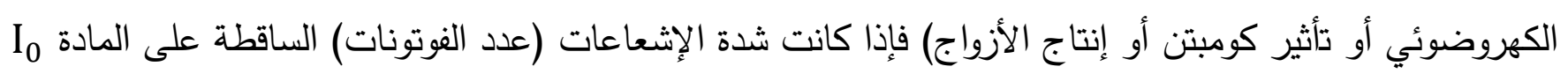

$$
\mathrm{I}=\mathrm{I}_{\mathrm{o}} \mathrm{e}^{-\mu \mathrm{x}} \quad \cdots \cdots \cdots
$$

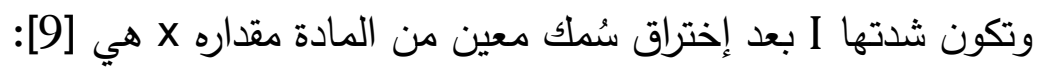

اذ إن بر: هو عبارة عن معامل التوهين الخطي لأشعة كاما.

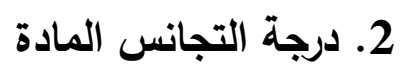

يمكن تحديد درجة تجانس السبيكة الأساس والمواد المتراكبة من خلال دراسة تباين شدة أشعة كاما ومعامل التوهين الخطي والانحراف المعياري.

3. تباين الثدة

يمكن معرفة تجانس المادة من خلال مقارنة شدة أشعة كاما I النافذة من المواقع المحددة في المادة مع معدل قيمها

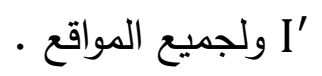

يمكن تحديد النسبة المئوية لتجانس المادة (1\% باستعمال العلاقة التالية [11] : $\ldots . . .(2) \Delta_{\mathrm{I}}(\%)=\frac{\mathrm{I}^{\prime}-\mathrm{I}}{\mathrm{I}^{\prime}} \times 100 \%$

4. تباين معامل التوهين الخطي يتم حساب معامل التوهين الخطي ب باستعمال العلاقة (1).

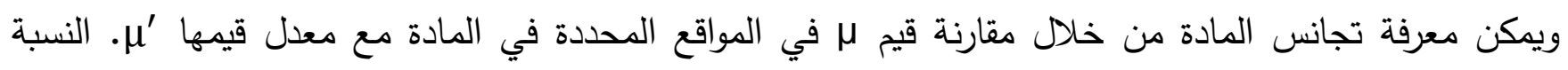

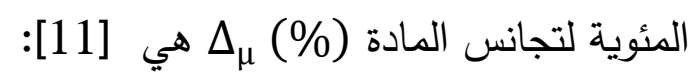
$\Delta_{\mu}(\%)=\frac{\mu^{\prime}-\mu}{\mu^{\prime}} \times 100 \%$

5. 5 الانحراف المعياري

يتم حساب الانحراف المعياري لمعامل التوهين (ر) م باستعمال العلاقة الآتية [11]: $(\mu)=\left[\Delta\left(\mu \mathrm{i}-\mu^{\prime}\right)^{2} /(\mathrm{N}-1)\right]^{0.5}$

إذ إن:

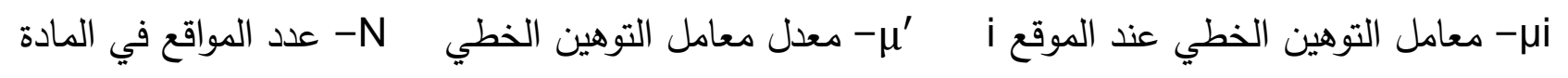
يمكن استعمال المعادلة (4) لحساب مقدار الانحراف المعياري لثدة أشعة كاما النافذة (ا)م. أما النسبة المئوية للإنحراف المعياري لكل من معامل التوهين الخطي وشدة أشعة كاما النافذة فتحتسب من خلال قسمة الإنحراف 


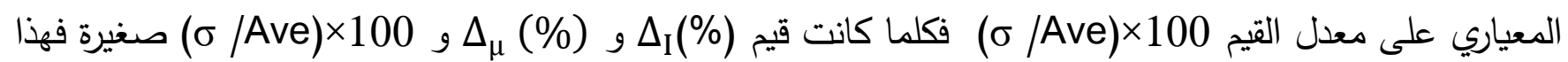
يعني أن المادة متجانسة (التباين قليل) [10]. النتائج والمناقشة المادة يوضح الجدول (1) أن معدل معامل التوهين الخطي يقل مع زيادة الطاقة وأن أعلى معدل لمعامل التوهين كان عند

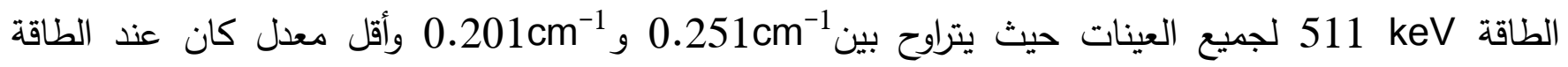
(1333 KeV) معامل التوهين الخطي كانت واضحة لكل عينة عند الطاقات الواطئة وتتقارب هذه القيم مع بعضها مع زيادة الطاقة

$$
\text { وتكون أكثر تقارباً عند الطاقات العالية. }
$$

الجدول (1): نتائج الدراسة الحالية لمعدل معامل التوهين الخطي والإنحراف المعياري والنسبة المئوية للانحراف المعياري ولطاقات مختلفة من أشعة كاما.

\begin{tabular}{|c|c|c|c|c|c|}
\hline \multicolumn{6}{|c|}{$\begin{array}{c}\mu \pm \sigma\left(\mathrm{cm}^{-1}\right) \\
((\sigma / \mu) \times 100(\%))\end{array}$} \\
\hline Sample & $511 \mathrm{keV}$ & $662 \mathrm{keV}$ & $1173 \mathrm{keV}$ & $1284 \mathrm{keV}$ & $1333 \mathrm{keV}$ \\
\hline $\mathrm{Al}-\mathrm{Co}-\mathrm{Ni}$ & $\begin{array}{c}0.251 \pm \\
0.008 \\
(3.3)\end{array}$ & $\begin{array}{c}0.212 \pm \\
0.002 \\
(1.1)\end{array}$ & $\begin{array}{c}0.113 \pm \\
0.006 \\
(5.2)\end{array}$ & $\begin{array}{c}0.089 \pm \\
0.004 \\
(4.5)\end{array}$ & $\begin{array}{c}0.079 \pm \\
0.006 \\
(7.3)\end{array}$ \\
\hline $\begin{array}{c}5 \% \underset{\mathrm{Cr}_{2} \mathrm{O}_{3}+\mathrm{Al}+}{\mathrm{Co}+\mathrm{Ni}} \\
\end{array}$ & $\begin{array}{c}0.252 \pm \\
0.005 \\
(2.4)\end{array}$ & $\begin{array}{c}0.183 \pm \\
0.005 \\
(2.9)\end{array}$ & $\begin{array}{c}0.111 \pm \\
0.004 \\
(3.2)\end{array}$ & $\begin{array}{c}0.085 \pm \\
0.002 \\
(2.3)\end{array}$ & $\begin{array}{c}0.077 \pm \\
0.006 \\
(7.8)\end{array}$ \\
\hline $\begin{array}{c}10 \% \mathrm{Cr}_{2} \mathrm{O}_{3}+\mathrm{Al}+ \\
\mathrm{Co}+\mathrm{Ni}\end{array}$ & $\begin{array}{c}0.251 \pm \\
0.006 \\
(2.4)\end{array}$ & $\begin{array}{c}0.210 \pm \\
0.004 \\
(2.0)\end{array}$ & $\begin{array}{c}0.125 \pm \\
0.004 \\
(3.2)\end{array}$ & $\begin{array}{c}0.086 \pm \\
0.004 \\
(4.4)\end{array}$ & $\begin{array}{c}0.081 \pm \\
0.004 \\
(5.1)\end{array}$ \\
\hline $\begin{array}{c}15 \% \underset{\mathrm{Co}}{\mathrm{Cr}_{2} \mathrm{O}_{3}}+\mathrm{Al}+ \\
\mathrm{Co}+\mathrm{Ni}\end{array}$ & $\begin{array}{c}0.201 \pm \\
0.008 \\
(4.0)\end{array}$ & $\begin{array}{c}0.184 \pm \\
0.004 \\
(2.2)\end{array}$ & $\begin{array}{c}0.086 \pm \\
0.004 \\
(4.8)\end{array}$ & $\begin{array}{c}0.062 \pm \\
0.004 \\
(6.4)\end{array}$ & $\begin{array}{c}0.062 \pm \\
0.003 \\
(4.8)\end{array}$ \\
\hline
\end{tabular}

رسمت العلاقة بين معدل معامل التوهين الخطي والطاقة لكل عينة وتبين أن العلاقة بينهما كانت خطية عكسية

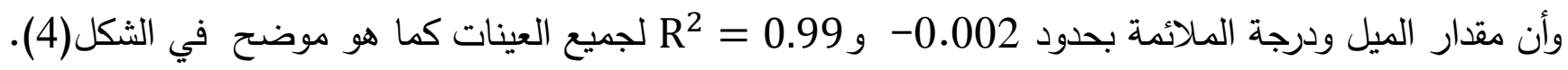
يمكن تحديد درجة تجانس السبيكة الأساس وتلك المدعمة بنسب مختلفة من مسحوق الكروم وذللك من خلال دراسة

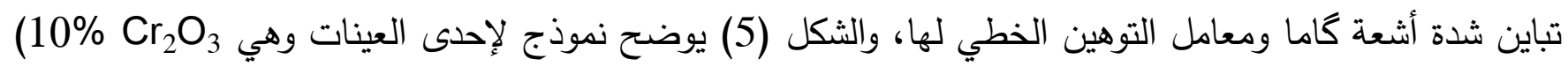


والتي كانت الأكثر تجانساً بين العينات الأخرى، ويتضح أن قيم (Al+ Co+Ni)

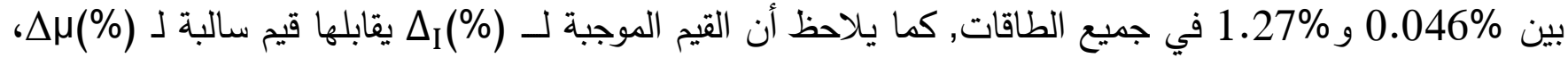
أي عند نقصان معامل التوهين الخطي يقابله زيادة في شدة الأشعة النافذة عند نفس المواقع والعكس صحيح أيضا.

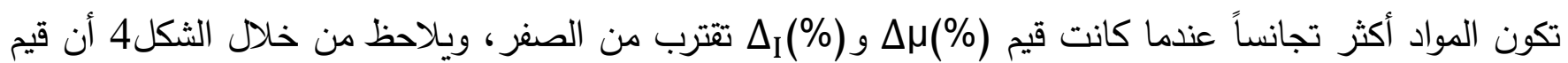

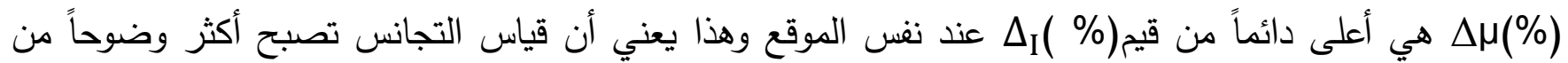

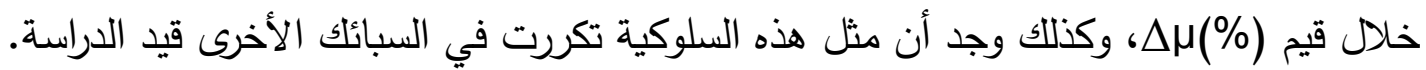
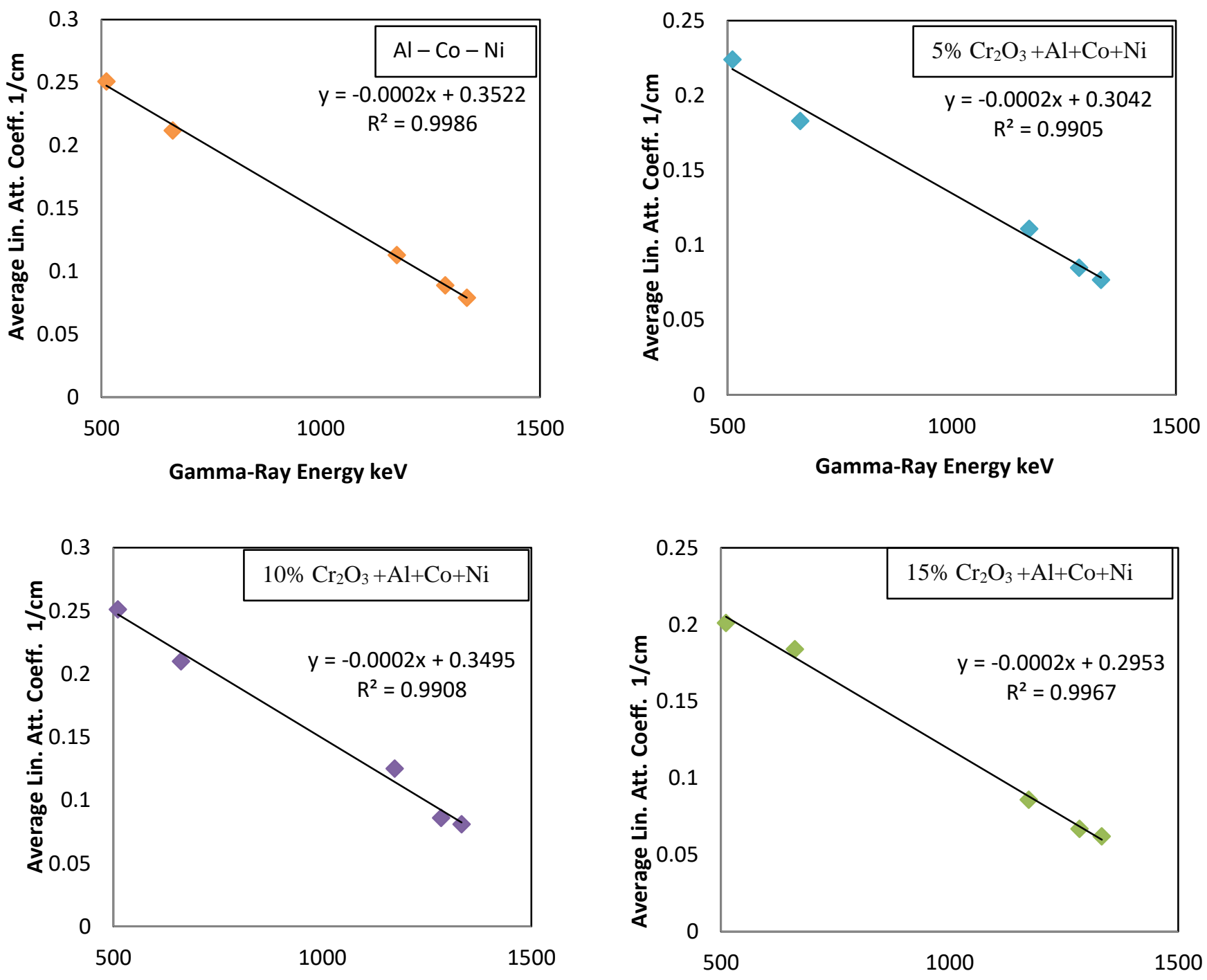

Gamma-Ray Energy keV

Gamma-Ray Energy keV

الثكل4. العلاقة الخطية بين الطاقة ومعدل معامل التو هين الخطي للعينات 

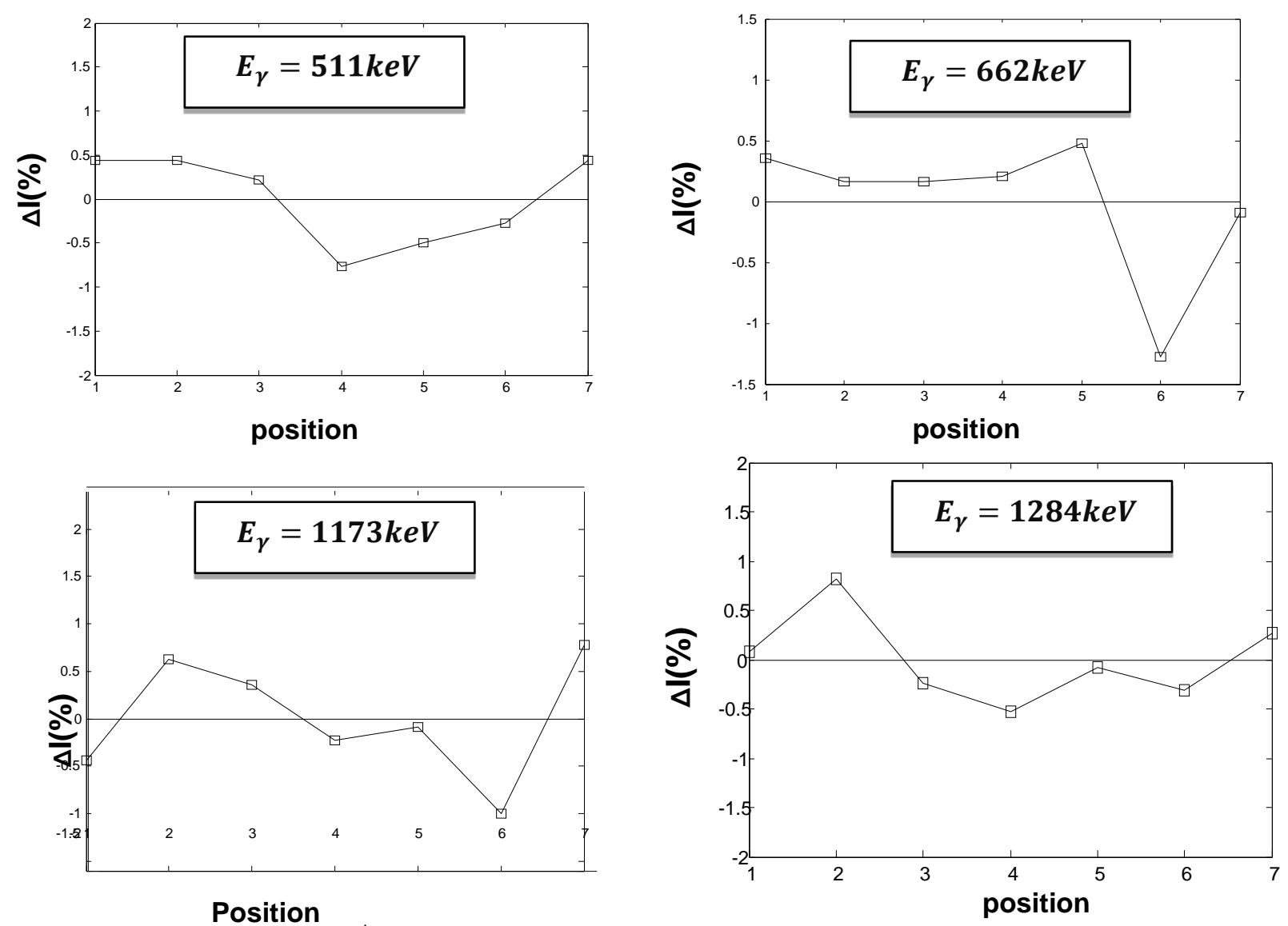

Position

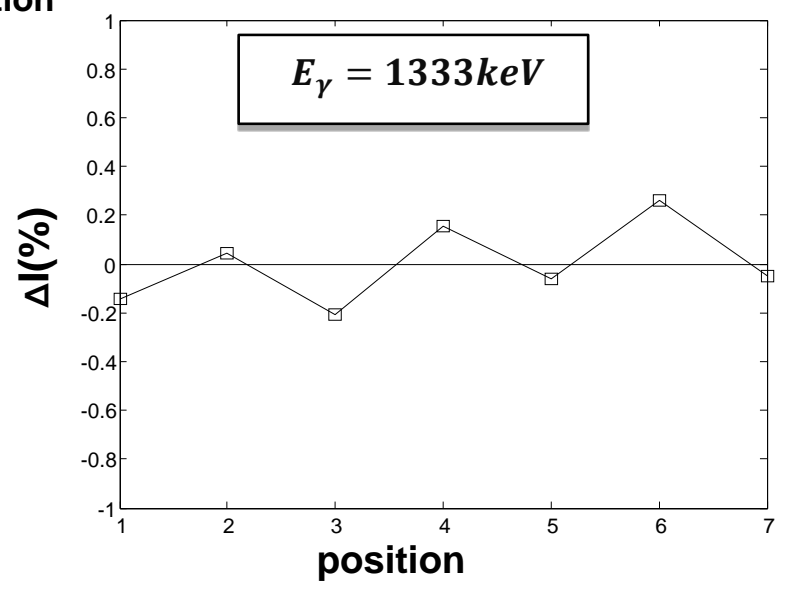

الثكل.5 يوضح النسبة المئوية للتباين في الثدة ومعامل التوهين مع الموقع للمادة المتراكبة قبل المعاملة

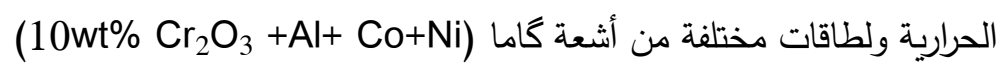


يبين كل من الثكل 6 والثكل 7 فحص الأشعة السينية XRD للعينة [5wt\% المجهري SEM وهذا تأكيد آخر على التجانس المنتظم للعينة.
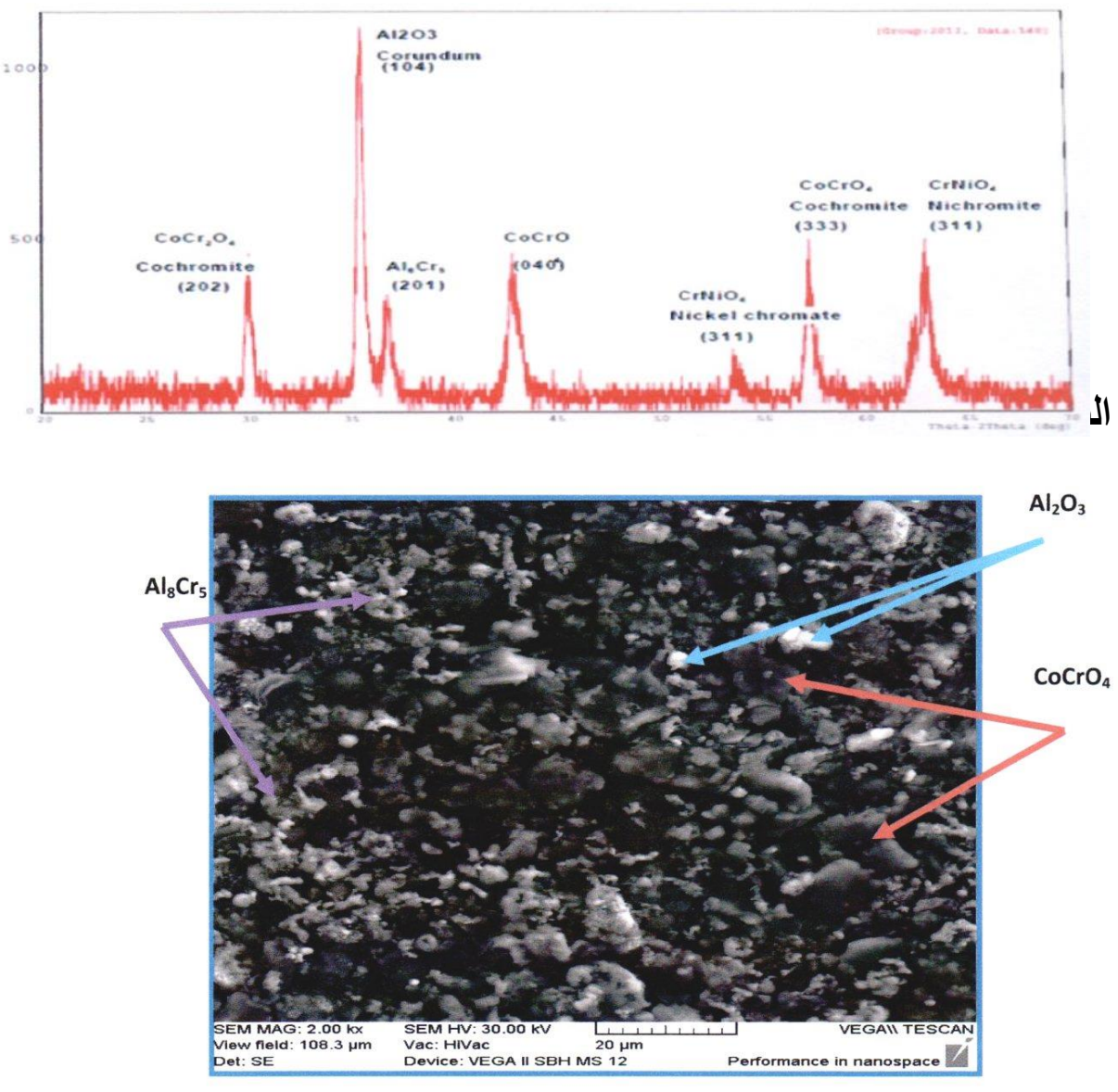

الثكل6. التصوير المجهري SEM للعينة ]

1- يمكن تحديد درجة تجانس السبيكة الأساس والمواد المتراكبة من خلال دراسة تباين شدة أشعة كاما ومعامل التوهين الخطي والإنحراف المعياري. 2- انخفضت قيم النسبة المئوية للتباين في الثدة ومعامل التوهين الخطي والانحراف المعياري للسبيكة الأساس والمادة المتراكبة $\left(\mathrm{Cr}_{2} \mathrm{O}_{3}+\mathrm{Al}+\mathrm{Co}+\mathrm{Ni}\right.$ عند الطاقات الواطئة مما يدل على أن المادة أصبحت أكثر تجانساً ولم يكن ذللك في الطاقات العالية للعينة بعد المعاملة الحرارية. 
3- أعطت قيم معدل معامل التوهين الخطي تبايناً واضحاً في الطاقات العالية في حين كانت القيم في الطاقات الواطئة متقاربة جداً.

4- بينت النتائج أن معدل معامل التوهين الخطي يتناسب عكسياً مع الطاقة وأن درجة الملائمة للعينات جميعها متساوية (0.99).

5- كانت النسبة المئوية للانحراف المعياري لمعامل التوهين الخطي للسبيكة الأساس والمواد المتراكبة منخفضة عند

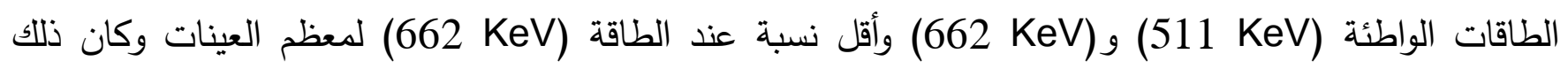
واضحاً حين أخذ المعدل لكل طاقة وللعينات جميعها وتكون اعلى القيم عند الطاقات العالية. أن أفضل دراسة للتجانس تكون عند الطاقة (662 KeV).

شكر وتقدير نود أن نعبر عن شكرنا وتقديرنا لمختبر بحوث الاغشية الرقيقة في قسم الفيزياء بكلية التربية للعلوم الصرفة في جامعة الموصل على توفير متطلبات البحث والتسهيلات المفيدة في مجال العمل. نئل

[1] M.M. Schwartz, "Composite Materials Hand Book” McGraw-Hill Book Company, 1984.

[2] R.J. Bruls, "The Thermal Conductivity of Magnetism Silicon Nitride $\left(\mathrm{MgSiN}_{2}\right)$ Ceramic and Related Materials", PhD thesis, Eindhoven University of Technology, 2000.

[3] A.C.Chaklader and K.R. Linklander and K.R. Linger,"Ceramic Metal Composites Using Metal Coated Aluminum Powder", Elsevier Composites, Vol.7, Issues 4, PP.239-243. 1976.

[4] S.B. Yousoff, "Effect of Milling Time on Mechanical and Physical Behavior of Al MMC Reinforced with $\mathrm{Al}_{2} \mathrm{O}_{3}$ ", Faculity of Mechanical Engineering, University Malysia Pahang, 2008.

[5] A.M. Al-Sariyah ,A. M. Farouk, A. B. Mohammed ,"Principles of Ionizing Radiation and Prevention". Kingdom of Saudi Arabia, King Saud University, Standing Committee for the Prevention of Radiation, 2007.

[6] M.M. Mohamed ,"Radiological Protection Principles and Applications", Amman, Jordan, 2005.

[7] K.Tibor and B. Róbert,"Aluminium Alloys Theory and Applications", $1^{\text {ed }}$, Printed in India, Published by InTech. 2011.

[8] W.D. Callistre, "Material Science and Engineering", 5 ${ }^{\text {th }}$ ed. U,S.A. Lilley J." Nuclear physics principles and application". Wiley, University of Manchester, New York. 2002

[9] S.J.Stankovic,R.D. Ilic. K. Jankovic, D. Bojovic and D.B. Loncar, "Gamma radiation absorption characteristics of concrete with components of different type metals", Acta Physica Polonica A Vol. 117, No. 5, 812-816. 2010

[10] L. Rabih, "The effect of DC-sputtering parameters and Al doping on conductivity and transparency of $\mathrm{TiO}_{2}$ thin films", doctoral dissertation. Gadjah Mada University, Yogyakarta, Indonesia, 2013.

[11] A.K. Mheemeed, I.H.Hasan, F.M. Al-Jomaily, "Gamma-ray absorption using rubber-lead mixtures as radiation protection shields". J Radioanal Nucl Chem., Vol. 291, No. 3,653-659. 2012 